\title{
Ultrastructural features of lymphocyte suppression induced by anthrax lethal toxin and treated with chloroquine
}

\author{
Mark I Hirsh', Irena Manov², Victoria Cohen-Kaplan ${ }^{3}$ and Theodore C lancu
}

Antibacterial therapy does not fully protect against anthrax because of severe systemic intoxication. Lysosomal processing of anthrax lethal toxin (LTX) is a key event in the disease pathogenesis, and agents interfering with this process, like chloroquine (CQ), may have practical applications. Although LTX is known to induce T-cell suppression, precise mechanisms of this phenomenon are not completely characterized. In the present study, we investigated alterations of lymphocyte ultrastructure caused by LTX and associated with favorable effect of CQ on the LTX-related dysfunction. Peripheral blood lymphocytes were activated via CD3 crosslinking in the presence or absence of LTX and CQ, and examined by transmission electron microscopy, flow cytometry and immunoblotting. Crosslinking of CD3 induced ultrastructural signs of lymphocyte activation, mostly disappeared after LTX treatment. The cell ultrastructure was well preserved in LTX-treated cells, despite dose- and time-dependent inhibition of T-cell function associated with impaired activation of mitogen-activated protein kinase. Regardless of intracellular signaling abnormalities, LTX did not decrease T-cell viability. CQ restored expression of CD69 $(P<0.001)$ and improved phosphorylation of p38 $(P=0.022)$ in LTXexposed T lymphocytes. The exposure of cells to CQ, with or without LTX, led to appearance of many phagolysosomes with heterogeneous content, possibly representing unprocessed internalized material. In conclusion, LTX suppressed Tcell functions, but did not affect the viability and caused no ultrastructural damage. Ultrastructural observations indicated that CQ reduced harmful effects of LTX, possibly by interfering with lysosomal activity.

Laboratory Investigation (2007) 87, 182-188. doi:10.1038/labinvest.3700505; published online 18 December 2006

KEYWORDS: anthrax; immunomodulation; signal transduction; T-cell activation; transmission electron microscopy

A lethal toxin (LTX) produced by B. anthracis consists of two associated proteins: protective antigen (PA) and lethal factor (LF). ${ }^{1}$ Susceptibility of various mammalian cells to harmful effects of anthrax toxins is associated with expression of cell surface receptors for PA. ${ }^{2}$ PA binds to these cell surface molecules and after proteolytic activation forms membranepenetrating heptamers capable of LF and edema toxin binding. ${ }^{1}$ The cell membrane-bound PA-LF complexes are internalized and after acidification in phagolysosomes dissociate resulting in translocation of LF into the cytosol. ${ }^{1,3}$ It has been shown that LF proteolytically cleaves several mitogen-activated protein kinase kinases (MAPKK), including MEK1/2, MKK3/4 and MKK6/7, and thereby disrupts regulation of major cellular functions by inhibition of intracellular signaling through MAPK cascades. ${ }^{4,5}$
Anthrax toxins derange the immune system facilitating progress of the infection. ${ }^{1,6,7}$ Specifically, LTX kills macrophages $(\mathrm{M} \phi)^{8,9}$ and inactivates the adaptive immune system. It has been demonstrated that LTX affects dendritic cell functions ${ }^{10,11}$ and therefore impairs antigen recognition and activation of $\mathrm{T}$ cells. Several in vitro reports have shown that LTX inhibits interleukin (IL)-2 production in Jurkat cells ${ }^{12}$ and interrupts signaling in human $\mathrm{T}$ cells. ${ }^{13,14}$ In addition, Comer et $a l^{15}$ have revealed the direct LTX-induced inactivation of $\mathrm{T}$ cells in vivo.

Currently recommended antibacterial therapy alone does not completely protect against anthrax because of the severe systemic intoxication ${ }^{6}$ and should be combined with other therapeutic approaches. One such approach may be affecting key steps in the development of intoxication, for instance

\footnotetext{
'Laboratory for Shock and Trauma Research, Ruth and Bruce Rappaport Faculty of Medicine, Technion, Haifa, Israel; ${ }^{2}$ Pediatric Research and Electron Microscopy Unit, Ruth and Bruce Rappaport Faculty of Medicine, Technion, Haifa, Israel and ${ }^{3}$ Cancer and Vascular Biology Research Center, Ruth and Bruce Rappaport Faculty of Medicine, Technion, Haifa, Israel

Correspondence: Dr MI Hirsh, MD, PhD, Laboratory for Shock and Trauma Research, Ruth and Bruce Rappaport Faculty of Medicine, POB 9649,31096 Haifa, Israel. E-mail: hirsh@tx.technion.ac.il

Received 31 August 2006; revised 16 October 2006; accepted 24 October 2006; published online 18 December 2006
} 
lysosomal processing of LTX. Chloroquine (CQ) is a pharmacological agent that interferes with lysosome functions and thereby disrupts this processing. CQ has protected isolated $\mathrm{M} \phi$ from deadly effects of LTX ${ }^{16,17}$ and even improved survival of LTX-treated mice. ${ }^{18}$ In light of these findings, we hypothesized that favorable effects of CQ were expanded beyond $\mathrm{M} \phi$, providing survival and/or functional benefits to other cell types. This assumption was further supported by demonstration of the ability of CQ to protect cultured epithelial cells from anthrax toxins $^{19}$ and to reduce suppression of T lymphocytes exposed to LTX. ${ }^{14}$ However, precise mechanisms of this protection are not completely understood. The present study was aimed to investigate alterations of lymphocyte ultrastructure caused by LTX and associated with favorable effect of CQ on the LTX-related dysfunction.

\section{MATERIALS AND METHODS Cell Culture}

Peripheral blood was obtained from healthy adult volunteers according the protocol approved by the Helsinki Committee of the Technion Faculty of Medicine. Peripheral blood mononuclear cells (PBMC) were isolated by centrifugation in the Histopaque 1077 (Sigma-Aldrich Co., Rehovot, Israel), and depleted from monocytes by adherence to plastic Petri dishes to separate peripheral blood lymphocytes (PBL). PBL fractions were more than $85 \%$ pure, and the cell viability was greater than $95 \%$ as determined by propidium iodide (PI, Sigma) staining and flow cytometry. Experiments were designed as described. ${ }^{14}$ Briefly, $10^{6} \mathrm{PBL}$ were resuspended in RPMI 1640 culture medium (Biological Industries, Kibbutz Beth Haemek, Israel) and incubated for different time periods at $37^{\circ} \mathrm{C}$ and $5 \% \mathrm{CO}_{2}$. Cells were activated by $\mathrm{CD} 3$ crosslinking with $1 \mu \mathrm{g} / \mathrm{ml}$ mouse anti-human CD3 monoclonal antibody (mAb; clone UCHT1) and 1:100 rabbit anti-mouse IgG (both from Serotec Ltd, Kidlington, UK). Recombinant PA, LF and mutant LF were purchased from List Biological Laboratories Inc. (Campbell, CA, USA). PA was added to the cells at concentration of $0.5 \mu \mathrm{g} / \mathrm{ml}$, whereas LF at concentrations of 10, 100, 1000 and $10000 \mathrm{ng} / \mathrm{ml}$. In part of experiments, CQ (Sigma) was used at concentration of $100 \mu \mathrm{M}$.

\section{Transmission Electron Microscopy}

Cell ultrastructure was assayed as described. ${ }^{20}$ Briefly, control and treated cells were fixed in $2 \%$ glutaraldehyde in $0.1 \mathrm{M}$ sodium cacodylate buffer, $\mathrm{pH} 7.2$, post-fixed in $2 \% \mathrm{OsO}_{4}$, dehydrated in ethanol series and embedded in epoxy. Ultrathin sections were stained with $1 \%$ uranyl acetate and $1 \%$ lead citrate, viewed and photographed with JEOL 100B (Tokyo, Japan) electron microscope operated at $60 \mathrm{kV}$. Three or more 300-mesh copper grids were examined from each sample in a blinded fashion.

\section{Flow Cytometry}

At the end of incubation, cells were stained with $\mathrm{mAb}$ against CD3 (clone UCHT1) and CD69 (clone CH/4, both from Serotec), rinsed and analyzed by flow cytometry (FACSCalibur ${ }^{\circledR}$; Becton Dickinson, Lincoln Park, NJ, USA). Percentages of double-positive cells within the lymphocyte gate and mean fluorescent intensity (MFI) were estimated, and an expression index was calculated by multiplying percentage of positive cells by MFI and expressed in arbitrary units (AU). The experiments were repeated six times with cells isolated from different donors. In parallel, autofluorescence control and relative isotypic controls (Serotec) were analyzed.

\section{Cell Viability}

LTX-induced T-cell apoptosis was assessed by triple staining with fluorescein isothiocyanate (FITC)-labeled anti-CD3 $\mathrm{mAb}$, cyanine 5 (Cy5)-labeled annexin $\mathrm{V}$ and PI, followed by flow cytometry. The procedure was carried out according to the instructions of the annexin $\mathrm{V}$ kit manufacturer (MBL Medical \& Biological Laboratories Co. Ltd, Nagoya, Japan) as described previously. ${ }^{20,21}$ The incidence of apoptotic T cells was expressed as percentage of $\mathrm{CD}^{+} /$annexin $\mathrm{V}^{+} / \mathrm{PI}^{-}$cells, whereas membrane-compromised (necrotic) $\mathrm{CD}^{+}$cells were $\mathrm{PI}^{+}$.

\section{Immunoblotting}

After the indicated treatments, cell extracts were prepared using protein extraction reagent (Pierce Biotechnology, Inc., Rockford, IL, USA), and aliquots of cell extract (30 $\mu \mathrm{g}$ of protein) were resolved by $10 \%$ polyacrylamide gel electrophoresis exactly as described. ${ }^{14}$ After electrophoresis and transfer, proteins were identified using a two-step chemiluminiscent detection (Pierce). Primary rabbit antibody against total and phosphorylated forms of p38 MAPK (Cell Signaling Technology, Inc., Beverly, MA, USA) was used. Films were analyzed by a Vilber Lourmat imaging system (Lyon, France) and the percentage of phosphorylated protein was calculated as fractions of total p38 protein. The experiments were repeated four times with cells isolated from different donors.

\section{Statistical Analysis}

Data are presented as mean \pm s.e.m. Comparison of the differences between groups (independent variables) was performed with one-way analysis of variance (ANOVA) followed by Neuman-Keuls post hoc test. $P$-values of $<0.05$ were considered statistically significant.

\section{RESULTS}

\section{Effect of LTX on Ultrastructure of Lymphocytes}

The LTX-induced suppression of lymphocyte functions has been amply documented. ${ }^{12-15}$ In the present study, we investigated alterations of lymphocyte ultrastructure associated with functional abnormalities induced by LTX. Crosslinking 

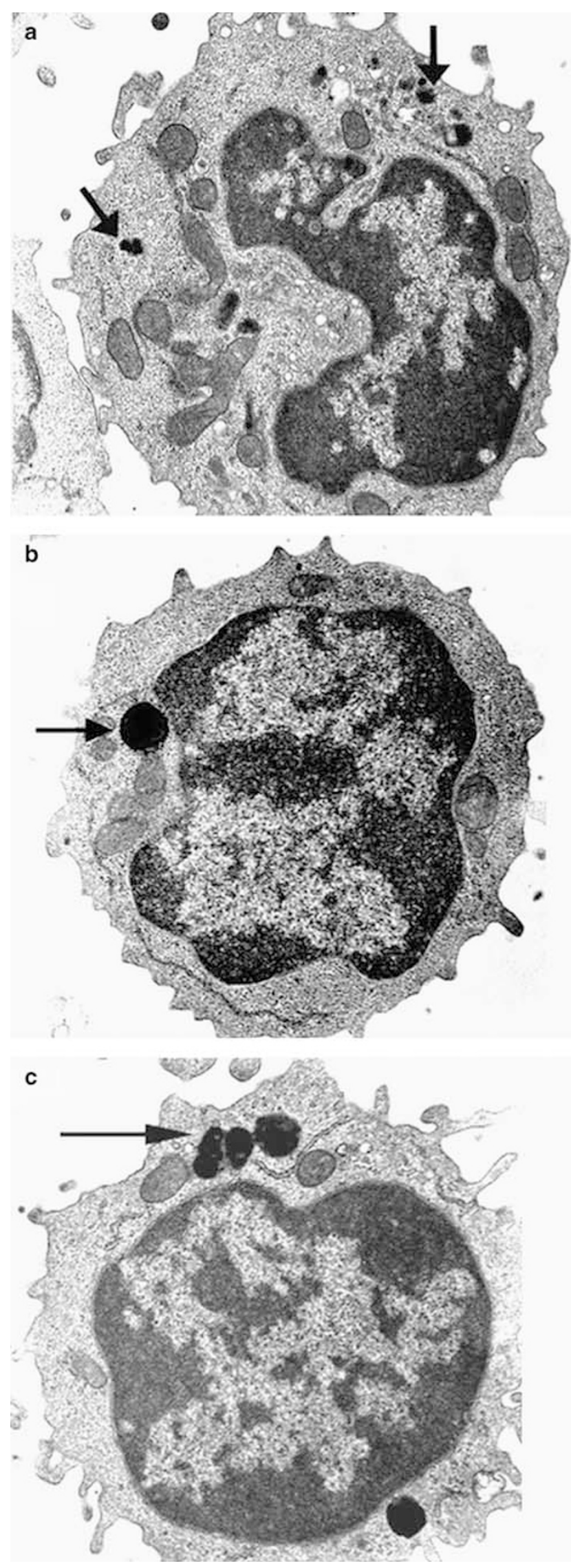

of CD3 markedly changed the ultrastructure of lymphocytes (Figure 1a). Nuclei frequently appeared indented and small electron lucent vacuoles, profiles of rough endoplasmic reticulum, free ribosomes and Golgi cisterns were present in the relatively wide rim of the cytoplasm. Electron-dense bodies, probably small lysosomes, were frequent and many mitochondria displayed variability of size and shape. In LTXtreated cells, ultrastructure was well preserved, and no evidence of organelle damage was found (Figure 1b). The cells usually showed reversal to the basic ultrastructure as observed before activation (untreated cells, Figure 1c): large, round- or oval-shaped nuclei and a narrow rim of cytoplasm containing few mitochondria, scant elements of endoplasmic reticulum and one or two electron-dense lysosomes were conspicuous. In addition, exposure of lymphocytes to LTX-containing mutant LF induced no cell damage; the cells remained well preserved and ultrastructurally resembled lymphocytes activated in the absence of LTX.

\section{Effect of LTX on Lymphocyte Functions}

Evaluation of the ability of $\mathrm{T}$ cells to undergo activation was in agreement with ultrastructural findings. The surface expression of CD69 on T cells was 6.5-fold lower following treatment with LTX $(P<0.0001)$ indicating severe impairment in lymphocyte activation (Figure 2a). Moreover, the LTX-impaired activation of T lymphocytes was apparently associated with abnormalities in intracellular signaling (Figure $2 \mathrm{~b}$ and $\mathrm{c}$ ). LTX initially reduced phosphorylation of p38 and by $30 \mathrm{~min}$ of exposure completely inhibited activation of this MAPK cascade (Figure $2 b$ and $c$ ). Signaling through p38 cascade remained inhibited over all the time that the cells had been exposed to LTX (data not shown). No effect of LTX was found on overall expression of p38 protein (Figure 2b). The LTX-induced inactivation of T cells was clearly dependent on LF concentrations (Figure 3a) and time of exposure (Figure 3b). Specificity of the LTX-induced effect was demonstrated by using a mutant LF, which was unable to cleave its cellular targets, ${ }^{4,5}$ and thus avoided decrease in CD69 expression (Figure 3a) and p38 phosphorylation

Figure 1 Ultrastructural appearance of activated human lymphocytes exposed to anthrax lethal toxin. A cell activated for $24 \mathrm{~h}$ by CD3 crosslinking (a) has an indented nucleus with marginated heterochromatin, wellpreserved microvilli and numerous mitochondria of variable size and shape. Electron-dense bodies, apparently small lysosomes, are frequent (arrows). In the center of the cell, transverse sections through Golgi cisterns and a few small electron-lucent vacuoles are visible. Treatment with LTX (b) reverses lymphocytes to the basic ultrastructure shown before activation: this cell appears similar to an untreated one, without signs of cell damage. A single electron-dense lysosome (arrow) is seen. An untreated cell (c) demonstrates the large nucleus, a few microvilli, and rare organelles in the cytoplasm: mitochondria, electron-dense lysosomes (arrows) and a few profiles of rough endoplasmic reticulum. TEM (uranyl acetate and lead citrate), original magnification, $\times 10000$. 
a
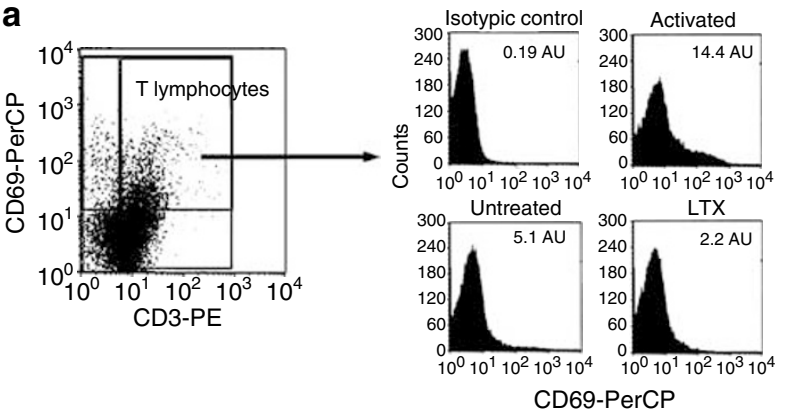

b

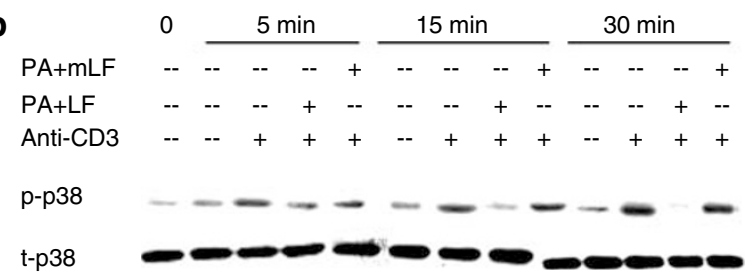

C

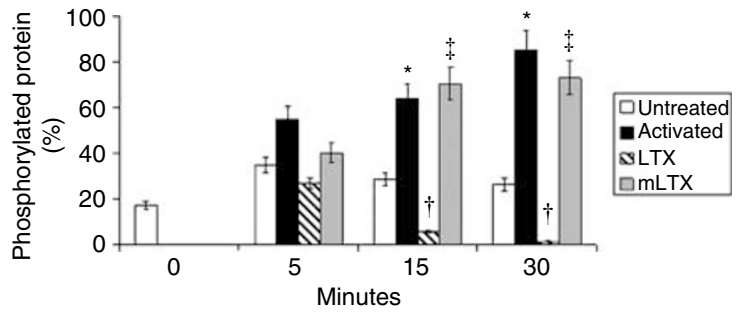

Figure 2 LTX suppresses functions of T lymphocytes. (a) Expression of CD69 on PBL activated for $24 \mathrm{~h}$ by CD3 crosslinking in the absence (activated) or presence of $0.5 \mu \mathrm{g} / \mathrm{ml} \mathrm{PA}$ and $0.5 \mu \mathrm{g} / \mathrm{ml}$ LF (LTX) was determined on $\mathrm{CD}^{+}$cells. In parallel, untreated cells, auto-fluorescence and relative isotypic controls were analyzed. An expression index calculated by multiplying of positive cells percentage by MFI was expressed in AU. Data from a representative experiment are shown $(n=6)$. Western blot (b) and densitometry data (c) represent the time course of p38 phosphorylation in PBL activated by $\mathrm{CD} 3$ crosslinking in the absence (activated) or presence of $0.5 \mu \mathrm{g} / \mathrm{ml} \mathrm{PA}$ and $0.5 \mu \mathrm{g} / \mathrm{ml}$ native or mutant LF (LTX or $\mathrm{mLTX}$, respectively). Untreated cells served as negative control. Percentages of phosphorylated protein were calculated as fractions of total p38 protein. Data from a representative experiment are shown $(n=4)$. ANOVA followed by Neuman-Keuls test: ${ }^{\star} P<0.05$ vs untreated; ${ }^{\dagger} P<0.015$ vs activated, ${ }^{\ddagger} P<0.006$ vs LTX.

(Figure $2 \mathrm{~b}$ and $\mathrm{c}$ ). No effect was found when PA or LF was separately added to the cells (data not shown).

\section{Effect of LTX on Lymphocyte Viability}

In order to study the effect of LTX on T-lymphocyte survival, we determined percentages of apoptotic (annexin V-positive) and necrotic (PI positive) $\mathrm{CD}^{+}$PBL. Despite the pronounced suppression of intracellular signaling, LTX did not increase the rate of T-cell apoptosis, which varied between 2.1 and $3.9 \%$ at different time points and LF concentrations (Figure 4). Only prolonged exposure to a very high LF concentration $(10 \mu \mathrm{g} / \mathrm{ml})$ increased apoptosis rates, but the increase was not statistically significant $(P=0.177)$, and was 2.9-folds lower than that induced by camptothecin
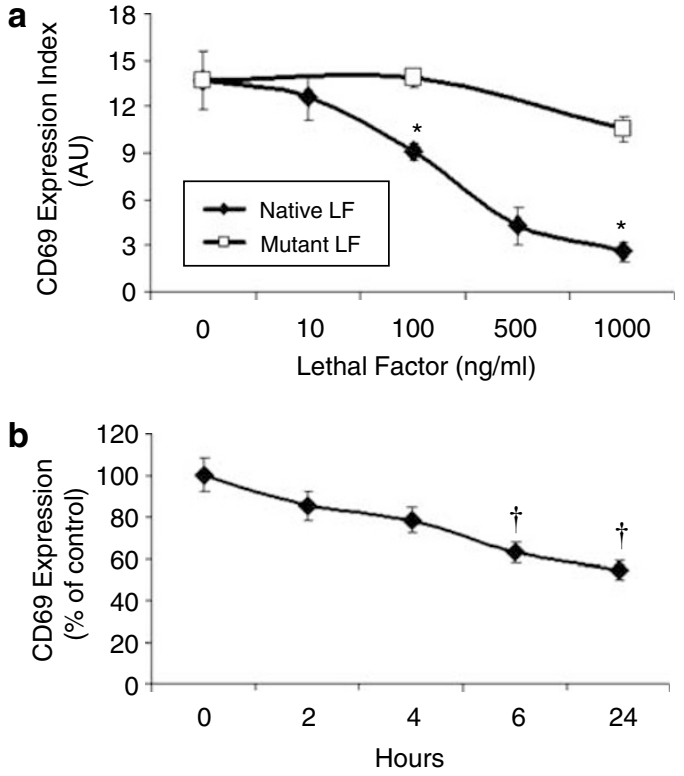

Figure 3 Effect of LTX on T-cell activation depends on concentration and time of exposure. (a) Expression of CD69 on CD3 ${ }^{+}$PBL activated for $24 \mathrm{~h}$ by CD3 crosslinking in the presence of $0.5 \mu \mathrm{g} / \mathrm{ml} \mathrm{PA}$ and the indicated concentrations of native or mutant LF. An expression index calculated by multiplying of positive cells percentage by MFI was expressed in AU. (b) The time course of $\mathrm{CD} 69$ expression on activated $\mathrm{CD} 3^{+} \mathrm{PBL}$ was determined at different time points after the exposure to $0.5 \mu \mathrm{g} / \mathrm{ml}$ of PA and $0.5 \mu \mathrm{g} / \mathrm{ml} \mathrm{LF}$. Expression of CD69 is presented as percentage of control (activated cells w/o LTX). Data represent results from four experiments. ANOVA followed by Neuman-Keuls test: ${ }^{\star} P<0.02$ vs mutant $L F ;{ }^{\dagger} P<0.005$ vs control.

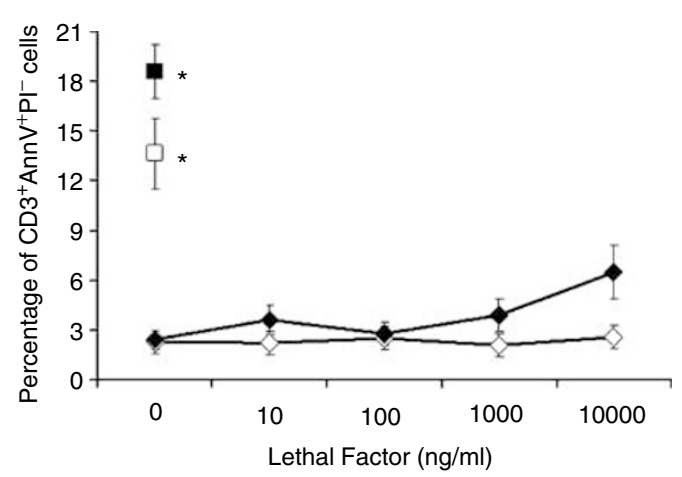

Figure 4 Anthrax lethal toxin does not increase apoptosis of $T$ lymphocytes. Binding of annexin $\mathrm{V}$ to $\mathrm{CD}^{+} \mathrm{PBL}$ exposed to $0.5 \mu \mathrm{g} / \mathrm{ml} \mathrm{PA}$ and the indicated concentrations of LF (diamonds) was determined after 6 (open symbols) and $24 \mathrm{~h}$ (filled symbols). Apoptotic T lymphocytes were identified as annexin $\mathrm{V}^{+} \mathrm{CD}^{+} \mathrm{PI}^{-}$. In parallel, untreated cells, autofluorescence and relative isotypic controls were analyzed. Cells exposed to $2 \mu \mathrm{g} / \mathrm{ml}$ camptothecin (Sigma) served as positive control (squares). Data represent results from four experiments. ANOVA followed by NeumanKeuls test: ${ }^{\star} P<0.01$ vs untreated cells (LF 0$)$.

$(18.6 \pm 1.6 \% ; P=0.01)$, a known apoptosis inducer ${ }^{22} \mathrm{em}-$ ployed as positive control (Figure 4). In addition, percentages of membrane-compromised (necrotic) cells were steady low at different concentrations of LTX and at different time points after exposure (data not shown). 


\section{Effect of CQ on Ultrastructure and Functions of Lymphocytes Treated with LTX}

Previous studies have shown that CQ protects isolated $\mathrm{M} \phi$ or T lymphocytes from LTX. ${ }^{14,16,17}$ Although when applied alone CQ had no substantial effect on T-cell activation, it restored the impaired expression of CD69 in T lymphocytes exposed to LTX (Figure 5a), and significantly improved phosphorylation of p38 (Figure 5b and c). We next investigated alterations of lymphocyte ultrastructure induced by CQ, and associated with its favorable effect on the LTXinduced dysfunction. Exposure of lymphocytes to CQ alone resulted in marked changes in cell ultrastructure (Figure 6a). Frequent single-membrane-limited bodies with heterogeneous content were detected within the cytoplasm. These bodies, apparently phagolysosomes or autophagolysosomes

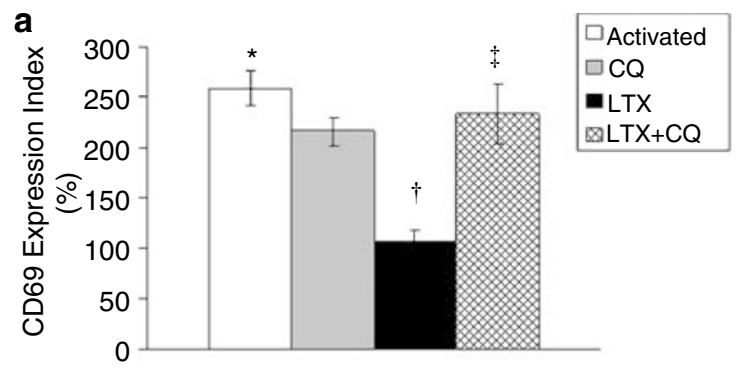

b
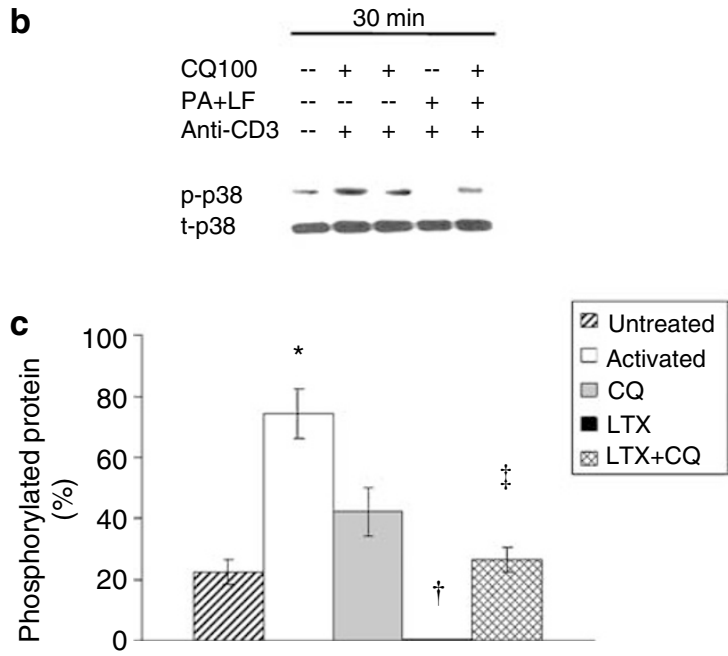

Figure 5 CQ protects T lymphocytes from LTX-induced suppression. (a) Flow cytometric analysis of CD69 expression on $\mathrm{CD} 3^{+} \mathrm{PBL}$ activated by $\mathrm{CD} 3$ crosslinking for $24 \mathrm{~h}$ in the absence (activated) or presence of $0.5 \mu \mathrm{g} / \mathrm{ml}$ PA and $0.5 \mu \mathrm{g} / \mathrm{ml} \mathrm{LF}$ (LTX), or $100 \mu \mathrm{M} \mathrm{CQ}$. Untreated cells served as negative control. An expression index was calculated by multiplying of positive cells percentage by MFI, and expressed as percentage of negative control. Data represent four experiments. Western blots (b) and densitometry data (c) of p38 phosphorylation in PBL activated by CD3 crosslinking for $30 \mathrm{~min}$ in the absence (activated) or presence of $0.5 \mathrm{PA}$ and $0.5 \mu \mathrm{g} / \mathrm{ml} \mathrm{LF}$ (LTX), and $100 \mu \mathrm{M}$ CQ. Untreated cells served as negative control. Percentages of phosphorylated protein were calculated as fraction of total p38 protein. Data from a representative experiment are shown $(n=4)$. ANOVA followed by Neuman-Keuls test: ${ }^{*} P<0.03$ vs untreated; ${ }^{\dagger} P<0.01$ vs activated, ${ }^{\ddagger} P<0.03$ vs LTX.
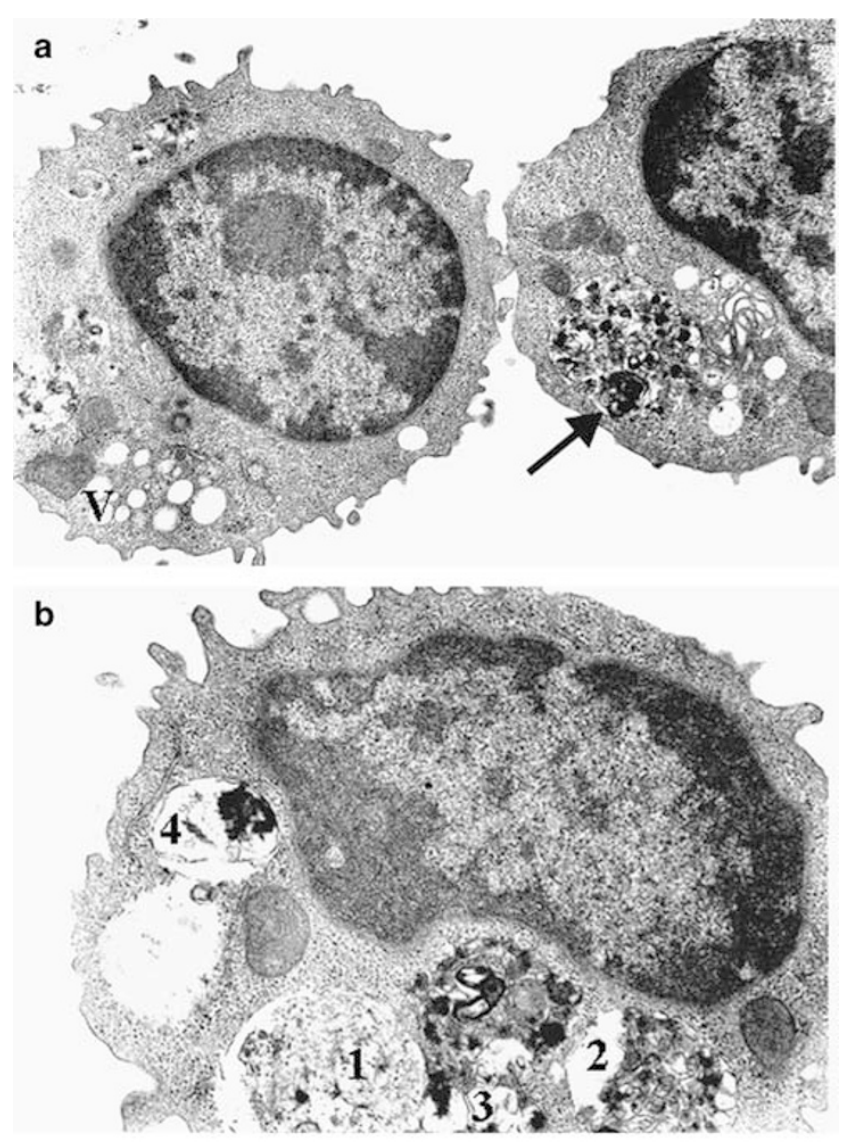

c

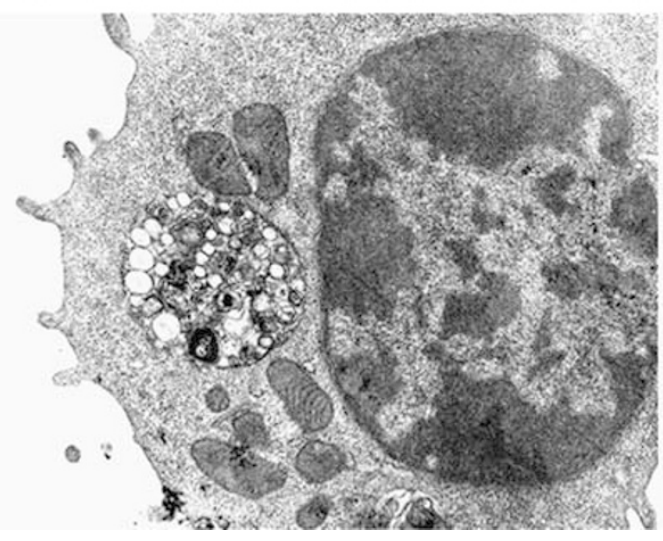

Figure 6 Ultrastructural appearance of activated human lymphocytes treated with CQ. Exposure to $\mathrm{CQ}$ alone (a) resulted in marked changes in ultrastructure: frequent single-membrane-bound bodies with heterogeneous content are noted in the cytoplasm (arrows). These bodies, apparently (auto) phagolysosomes contain membranous material, possibly representing unprocessed engulfed particles and/or cell debris. A number of electron lucent vesicles $(\mathrm{V})$ and fat droplets are also seen. TEM (uranyl acetate and lead citrate), original magnification, $\times 8000$. Concomitant treatment with LTX and CQ (b) revealed no additional damage to the cell. Numerous lysosomes with abundant amorphous (1) or membranous (2, 3 and 4$)$ content are conspicuous. Microvilli and mitochondria are well preserved. The nucleus shows a mild indentation. TEM (uranyl acetate and lead citrate), original magnification, $\times 15000$. Occasional cells (c) appear near-normal showing a single multivesicular body, normal mitochondria and microvilli, and some clumping of heterochromatin adjacent to the nuclear membrane. TEM (uranyl acetate and lead citrate), original magnification, $\times 10000$. 
contained abundant segregated compounds and/or cell debris. Additionally, a number of electron lucent vesicles and fat droplets were seen. Cells concomitantly treated with LTX and CQ showed similar ultrastructural changes. Nuclei were mildly indented, microvilli and mitochondria appeared preserved. In the cytoplasm, many lysosomes with various amorphous or membranous contents were observed (Figure $6 \mathrm{~b}$ and $\mathrm{c})$.

\section{DISCUSSION}

In the present study, LTX impaired T-lymphocyte functions in a dose- and time-dependent manner, but not their viability, and did not induce structural damage. The main finding was ultrastructural evidence that CQ protected T-cells from LTX, possibly by interfering with lysosome activity.

Unlike $\mathrm{M} \phi$, which could be easily killed by anthrax toxins. ${ }^{8,9,23}$ LTX did not affect the viability of T lymphocytes in either the present study or a previous report. ${ }^{13}$ This phenomenon can be explained by the involvement of numerous independent systems, such as MAPK, PI3-Akt, JAK/STAT, PKC $\theta$ and others in T-cell survival control. ${ }^{24,25}$ Thus, it is reasonable that parallel mechanisms hold the cell survival under control even when MAPK has been inactivated. However, the effects of LTX on signaling through other than MAPK regulatory pathways are still unknown and further efforts are requested to clarify this issue.

In concordance with determination of viability by flow cytometry, no signs indicating cellular damage were found using TEM. When CD3-crosslinked lymphocytes were treated with LTX, the ultrastructural features of untreated normal lymphocytes reappeared. Ferrarini et $a l^{26}$ have described a subset of $\mathrm{T}$ lymphocytes in peripheral human blood, which display ultrastructural features identical to those of activated lymphocytes as seen in our study (Figure 1a). Details of the method of cell purification and functional properties of isolated cells, primarily the expression of high-affinity receptors to IgG (CD64 by the current classification), strongly suggest that the authors observed activated lymphocytes. In summary, CD3 crosslinking produced ultrastructural features of lymphocyte activation, which were abrogated in the presence of LTX.

In contrast to the preserved ultrastructure and viability, LTX inhibited activation of $\mathrm{T}$ lymphocytes manifested by expression of CD69 in a dose- and time-dependent manner. This is consistent with our previous report and data of other investigators who have determined expression of CD69 and cytokines in LTX-treated lymphocytes. ${ }^{12-15}$ The novel information gathered during this study is characterization of cell ultrastructure changes associated with severe T-lymphocyte suppression in anthrax, as well as comparison of ultrastructural findings with functional tests (viability, activation, intracellular signaling).

The impaired T-lymphocyte activation was expectedly associated with MAPK inhibition. Both p38 (Figure 2) and ERK $1 / 2^{14}$ cascades were inactivated during first $30 \mathrm{~min}$, and remained inhibited over all the time $(24 \mathrm{~h})$ that the cells had been exposed to LTX. The time-dependent inhibition of MAPK phosphorylation is fully consistent with data of other investigators that have shown similar suppressive effect of LTX on MAPK in $\mathrm{M} \phi,{ }^{5,23}$ dendritic cells, ${ }^{10,11}$ Jurkat cells and normal lymphocytes. ${ }^{12-15}$ Specific character of these abnormalities was demonstrated using the mutant form of LF (Figure $2 \mathrm{~b}$ and $\mathrm{c}$ ), which was unable to cleave its intracellular targets owing to a point mutation. ${ }^{4,5}$

CQ is an antimalarial agent, also used for treatment of chronic inflammatory and autoimmune diseases. ${ }^{27}$ Mechanisms of antiinflammatory effects of CQ are not completely clear; most probably CQ interferes with presentation and recognition of antigens by blocking of lysosomal processing of major histocompatibility complex molecules. ${ }^{27}$ An ability of CQ to inhibit proton pumps, and therefore disrupt proton gradients leading to lysosome $\mathrm{pH}$ decrease, has been amply documented. ${ }^{27,28}$ Acidification of lysosomes is a key event in the pathogenesis of anthrax, being essential for dissociation of internalized PA-LF complexes. Hence, agents interfering with this process may have practical applications. In fact, no dramatic effect of CQ per se on T-cell functions was observed. However, an apparently inhibiting effect on lysosome functioning was documented using TEM. Taken together, the finding of frequent lysosomes with heterogeneous content, in parallel with other well-preserved subcellular elements and organelles, suggests a selective damage to the processing of internalized and lysosome-compartmentalized material. These findings correspond well with the antilysosomal activity of CQ demonstrated biochemically, ${ }^{16,19,29}$ and are consistent with data on the effect of inhibitors of lysosomal acidification on ultrastructure and functions of T-cell lysosomes. ${ }^{30}$

CQ has protected $\mathrm{M} \phi,{ }^{16,17}$ epithelial cells ${ }^{19}$ or T lymphocytes ${ }^{14}$ from LTX, and even increased survival of LTX-treated mice. ${ }^{18}$ In LTX-exposed lymphocytes, CQ improved phosphorylation of $\mathrm{p} 38$, and recovered transduction of the intracellular signals resulting in enhanced CD69 expression (Figure 5). Most probably, these favorable effects of CQ were related to its inhibitory lysosomotropic activity (Figure 6). It seems quite reasonable to propose that the impaired lysosome acidification prevents dissociation of internalized LF-PA complexes, and thereby avoids or greatly reduces the exposure of MAPKKs to enzymatically active LF. This suggestion is supported by the ultrastructural evidence of retention of abundant material in the lysosome compartment of lymphocytes. Our ultrastructural findings are similar to the previously described CQ-induced lysosome disorder, featured as appearance of large, foamy or granular autophagolysosomes; or collection of membranous vesicles in the lysosome (multivesicular body), subsequent to the progressive accumulation of cell membrane aggregates and myeloid bodies owing failure of enzymatic digestion. ${ }^{31}$

In conclusion, our findings are the first to demonstrate that, despite the profound impairment of T-lymphocyte functions, LTX does not induce ultrastructural damage and 
does not affect cell viability. The favorable effect of CQ reducing the LTX-induced T-cell suppression is associated with ultrastructural evidence of abnormal lysosomal functioning.

\section{ACKNOWLEDGEMENT}

We do not have commercial or other associations that might pose a conflict of interest. This study was supported in part by the Dan David Foundation (Grant 005/IM/5C)

1. Collier RJ, Young JA. Anthrax toxin. Annu Rev Cell Dev Biol 2003;19: 45-70.

2. Bradley KA, Mogridge J, Mourez M, et al. Anthrax delivers a lethal blow to host immunity. Nat Med 2003;9:996-997.

3. Ascenzi PP, Visca P, Ippolito G, et al. Anthrax toxin: a tripartite lethal combination. FEBS Let 2002;531:384-388.

4. Duesbery NS, Webb $\mathrm{CP}$, Leppa $\mathrm{SH}$, et al. Proteolytic inactivation of MAP-kinase-kinase by anthrax lethal factor. Science 1998;280:734737.

5. Vitale G, Pellizzari R, Recchi $C$, et al. Anthrax lethal factor cleaves the $\mathrm{N}$-terminus of MAPKKs and induces tyrosine/threonine phosphorylation of MAPKs in cultured macrophages. Biochem Biophys Res Commun 1998:248:706-711.

6. Inglesby TV, O'Toole T, Henderson DA, et al. Anthrax as a biological weapon, 2002: updated recommendations for management. JAMA 2002;287:2236-2252.

7. Dixon TC, Meselson M, Guillemin J, et al. Anthrax. N Engl J Med 1999;341:815-826

8. Agrawal A, Pulendran B. Anthrax lethal toxin: a weapon of multisystem destruction. Cell Mol Life Sci 2004;61:2859-2865.

9. Hanna PC, Acosta D, Collier RJ. On the role of macrophages in anthrax. Proc Natl Acad Sci USA 1993;90:10198-10201.

10. Agrawal A, Lingappa J, Leppa SH, et al. Impairment of dendritic cells and adaptive immunity by anthrax lethal toxin. Nature 2003;424: 329-334.

11. Tournier J-N, Quesnel-Hellmann A, Mathieu J, et al. Anthrax edema toxin cooperates with lethal toxin to impair cytokine secretion during infection of dendritic cells. J Immunol 2005;174:4934-4941.

12. Fang $\mathrm{H}$, Cordoba-Rodriguez $\mathrm{R}$, Lankford CSR, et al. Anthrax lethal toxin blocks MAPK kinase-dependent IL-2 production in CD4+ T cells. J Immunol 2005;174:4966-4971.

13. Paccani SR, Tonello F, Ghittoni R, et al. Anthrax lethal toxins suppress $T$ lymphocyte activation by disrupting antigen receptor signaling. J Exp Med 2005;201:325-331.

14. Hirsh MI, Cohen V. Chloroquine prevents T lymphocyte suppression induced by anthrax lethal toxin. J Infect Dis 2006;194:1003-1007.

15. Comer JE, Chopra AK, Peterson JW, et al. Direct inhibition of Tlymphocyte activation by anthrax toxins in vivo. Infect Immun 2005;73:8275-8281.
16. Friedlander AM. Macrophages are sensitive to anthrax lethal toxin through an acid-dependent process. J Biol Chem 1986;261:7123-7126.

17. Komiyama T, Swanson JA, Fuller RS. Protection from anthrax toxinmediated killing of macrophages by the combined effects of furin inhibitors and chloroquine. Antimicrob Agents Chemother 2005;49:3875-3882.

18. Artenstein AW, Opal SM, Cristofaro $P$, et al. Chloroquine enhances survival in B. anthracis intoxication. J Infect Dis 2004;190:1655-1660.

19. Gordon VM, Leppla SH, Hewlett EL. Inhibitors of receptor-mediated endocytosis block the entry of Bacillus anthracis adenylate cyclase toxin but not that of Bordetella pertussis adenylate cyclase toxin. Infect Immun 1988;56:1066-1069.

20. Manov I, Hirsh M, lancu TC. N-acetylcysteine does not protect HepG2 cells against acetaminophen-induced apoptosis. Pharmacol Toxicol 2004;94:213-225.

21. Hirsh M, Kaplan V, Dyugovskaya L, et al. Response of lung NK1.1positive natural killer cells to experimental sepsis in mice. Shock 2004:22:40-45.

22. Span LF, Pennings AH, Vierwinden $G$, et al. The dynamic process of apoptosis analyzed by flow cytometry using Annexin-V/propidium iodide and a modified in situ end labeling technique. Cytometry 2002:47:24-31.

23. Park JM, Greten FR, Li ZW, et al. Macrophage apoptosis by anthrax lethal factor through p38 MAP kinase inhibition. Science 2002;297:2048-2051.

24. Benczik M, Gaffen SL. The interleukin (IL)-2 family cytokines: survival and proliferation signaling pathways in T lymphocytes. Immunol Invest 2004;33:109-142.

25. Altman A, Isakov N, Baier G. Protein kinase C $\theta$ : a new essential superstar on the T cell stage. Immunol Today 2000;21:567-573.

26. Ferrarini M, Cadoni A, Franzi AT, et al. Ultrastructure and cytochemistry of human peripheral blood lymphocytes. Similarities between the cells of the third population and TG lymphocytes. Eur J Immunol 1980;10:562-570.

27. O'Neill PM, Bray PG, Hawley SR, et al. 4-Amino-quinolines-past, present and future: a chemical perspective. Pharmacol Ther 1998:77:29-58.

28. Lujken JJ, Aerts JM, Mejer AJ. The role of the intralysosomal $\mathrm{pH}$ in the control of autophagic proteolytic flux in rat hepatocytes. Eur J Biochem 1996;235:564-573.

29. Schneider P, Korolenko TA, Busch U. A review of drug-induced lysosomal disorders of the liver in man and laboratory animals. Microsc Res Tech 1997;36:253-275.

30. Kataoka T, Takaku K, Magae J, et al. Acidification is essential for maintaining the structure and function of lytic granules of CTL. Effect of concanamycin A, an inhibitor of vacuolar type H+-ATPase, on CTL-mediated cytotoxicity. J Immunol 1994;153:3938-3947.

31. Cheville NF. Ultrastructural Pathology. An Introduction to Interpretation. lowa State University Press: Ames, IA, 1994, pp $147-148$. 